\title{
Capacity and Optimal Collusion Attack Channels for Gaussian Fingerprinting Games
}

\author{
Ying Wang and Pierre Moulin ${ }^{a}$ \\ ${ }^{a}$ Beckman Institute, Coordinated Science Lab \\ and Department of Electrical and Computer Engineering \\ University of Illinois at Urbana-Champaign \\ Urbana, IL 61801 USA
}

\section{INTRODUCTION}

In content fingerprinting, the same media covertext - image, video, audio, or text - is distributed to many users. A fingerprint, a mark unique to each user, is embedded into each copy of the distributed covertext. In a collusion attack, two or more users may combine their copies in an attempt to "remove" their fingerprints and forge a pirated copy. To trace the forgery back to members of the coalition, we need fingerprinting codes that can reliably identify the fingerprints of those members. Researchers have been focusing on designing or testing fingerprints for Gaussian host signals and the mean square error (MSE) distortion under some classes of collusion attacks, in terms of the detector's error probability in detecting collusion members. For example, under the assumptions of Gaussian fingerprints and Gaussian attacks (the fingerprinted signals are averaged and then the result is passed through a Gaussian test channel), Moulin and Briassouli ${ }^{1}$ derived optimal strategies in a game-theoretic framework that uses the detector's error probability as the performance measure for a binary decision problem (whether a user participates in the collusion attack or not); Stone ${ }^{2}$ and Zhao et al. ${ }^{3}$ studied average and other non-linear collusion attacks for Gaussian-like fingerprints; Wang et al. ${ }^{4}$ stated that the average collusion attack is the most efficient one for orthogonal fingerprints; Kiyavash and Moulin ${ }^{5}$ derived a mathematical proof of the optimality of the average collusion attack under some assumptions.

In this paper, we also consider Gaussian cover signals, the MSE distortion, and memoryless collusion attacks. We do not make any assumption about the fingerprinting codes used other than an embedding distortion constraint. Also, our only assumptions about the attack channel are an expected distortion constraint, a memoryless constraint, and a fairness constraint. That is, the colluders are allowed to use any arbitrary nonlinear strategy subject to the above constraints. Under those constraints on the fingerprint embedder and the colluders, fingerprinting capacity is obtained as the solution of a mutual-information game involving probability density functions (pdf's) designed by the embedder and the colluders. We show that the optimal fingerprinting strategy is a Gaussian test channel where the fingerprinted signal is the sum of an attenuated version of the cover signal plus a Gaussian information-bearing noise, and the optimal collusion strategy is to average fingerprinted signals possessed by all the colluders and pass the averaged copy through a Gaussian test channel. The capacity result and the optimal strategies are the same for both the private and public games. In the former scenario, the original covertext is available to the decoder, while in the latter setup, the original covertext is available to the encoder but not to the decoder.

\section{STATEMENT OF THE PROBLEM}

Both of the private and public fingerprinting setups are modelled in Fig. 1. Let the covertext sequence $\mathbf{S}=$ $\left(S_{1}, \ldots, S_{N}\right)$ consist of independent and identically distributed (i.i.d.) samples drawn from a Gaussian pdf $\mathcal{N}\left(0, \sigma^{2}\right)$. The fingerprinting encoder generates $|\mathcal{M}|=\left\lceil 2^{N R}\right\rceil$ fingerprinted copies $\mathbf{X}_{m}=f_{N}(\mathbf{S}, m)$, where $f_{N}: \mathbb{R}^{N} \times \mathcal{M} \rightarrow \mathbb{R}^{N}$ is the fingerprinting code and $R$ is its rate. Each fingerprint $m$ corresponds to a user. Assume that the collusion size is $L=2 .^{*}$ Let $M_{1}$ and $M_{2}$ be two users selected independently and uniformly from the set $\mathcal{M}$. They collude to produce a pirated copy, or forgery, $\mathbf{Y} \in \mathbb{R}^{N}$, through an attack channel $p_{\mathbf{Y} \mid \mathbf{X}_{1} \mathbf{X}_{2}}$. The decoder does not know $p_{\mathbf{Y} \mid \mathbf{X}_{1} \mathbf{X}_{2}}$ selected by the two colluders. The decoder produces estimates $\left(\hat{M}_{1}, \hat{M}_{2}\right)=g_{N}(\mathbf{Y}) \in \mathcal{M}^{2}$ in attempt to catch the two colluders.

\footnotetext{
${ }^{*}$ In this paper, we focus on the case of two colluders for the simplicity of notation. Although cumbersome, it is conceptually straightforward to extend to the case where there are more than two colluders.
} 


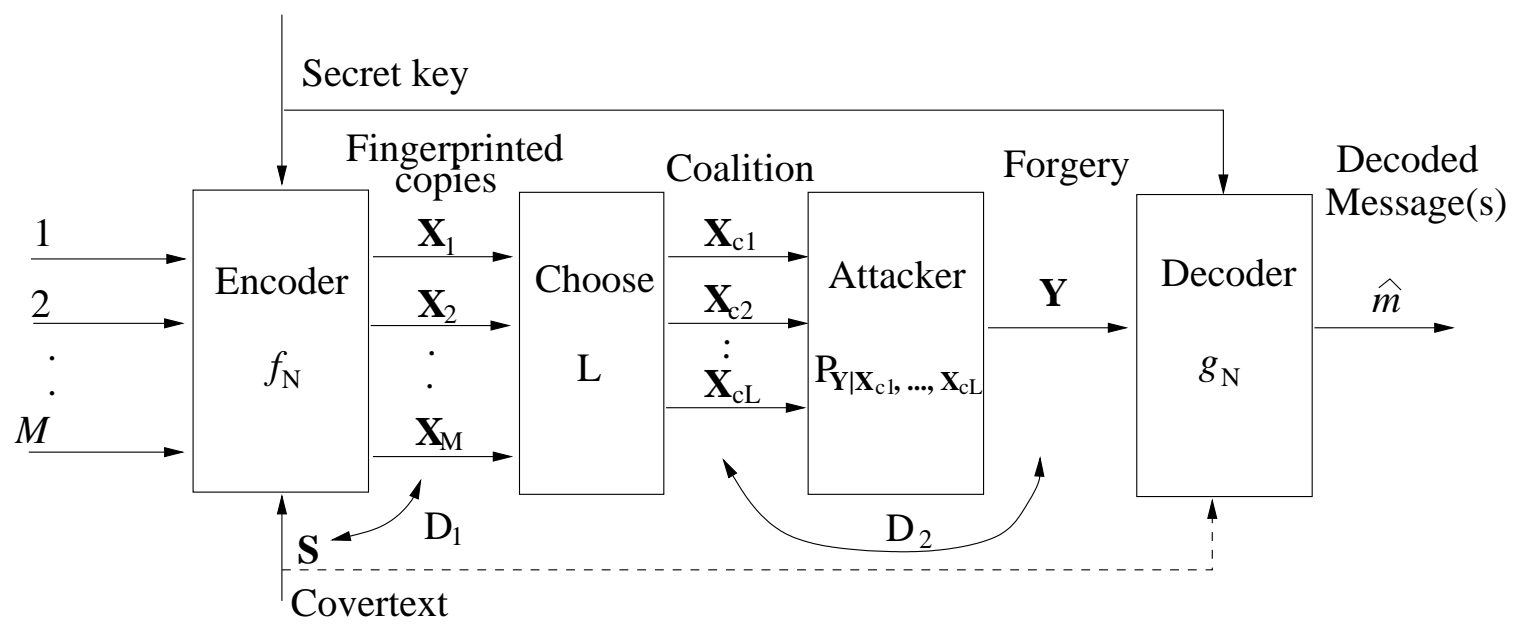

Figure 1. Fingerprinting games.

\subsection{Notation}

We use uppercase letters for random variables, lowercase letters for their individual values, and boldface fonts for sequences (or vectors). Differential entropy of a random variable $X$ is denoted by $h(X)$, and the mutual information between two random variables $X$ and $Y$ is denoted by $I(X ; Y)$. The mathematical expectation is denoted by $\mathrm{E}$.

\subsection{Constrained Fingerprint Codes and Collusion Attack Channels}

The MSE distortion between a fingerprinted signal sequence $\mathbf{x}$ and a covertext signal sequence $\mathbf{s}$ is defined as

$$
d^{N}(\mathbf{s}, \mathbf{x})=\frac{1}{N} \sum_{i=1}^{N}\left(x_{i}-s_{i}\right)^{2} .
$$

Fingerprinting codes are required to satisfy

$$
\mathrm{E} d^{N}(\mathbf{S}, \mathbf{X}) \leq D_{1}
$$

where $D_{1} \geq 0$.

DeFINITION 2.1. A covert private fingerprinting channel is a conditional pdf $p_{X \mid S}(x \mid s)$ such that

$$
\sum_{s, x} p_{X \mid S}(x \mid s) p_{S}(s)(x-s)^{2} \leq D_{1}
$$

We denote by $\mathcal{Q}_{1}$ the set of such feasible covert private fingerprinting channels.

DeFINITION 2.2. Given an auxiliary random variable $U \in \mathbb{R}$, a covert public fingerprinting channel is a conditional pdf $p_{X U \mid S}(x, u \mid s)$ such that

$$
\sum_{u, s, x} p_{X U \mid S}(x, u \mid s) p_{S}(s)(x-s)^{2} \leq D_{1}
$$

We denote by $\mathcal{Q}_{2}$ the set of such feasible covert public fingerprinting channels.

We define a constrained discrete memoryless collusion channel (DMCC) as a pdf $p_{Y \mid X_{1} X_{2}}$ subject to a fairness constraint

$$
p_{Y \mid X_{1} X_{2}}=p_{Y \mid X_{2} X_{1}}
$$


and the expected MSE distortion constraint

$$
\mathrm{E} d^{N}(\mathbf{X}, \mathbf{Y}) \leq D_{2}
$$

We pose the fairness constraint and the $D_{2}$ constraint on both colluders, based on the assumption that neither colluder is willing to take more risks than the other colluder. If this assumption does not hold, then any attempt to catch all colluders is futile because one colluder can make negligible or even zero contribution to the pirated copy $\mathbf{Y}$.

Definition 2.3. A discrete memoryless collusion attack channel is a conditional pdf $p_{Y \mid X_{1} X_{2}}\left(y \mid x_{1}, x_{2}\right)$ such that

$$
p_{Y \mid X_{1} X_{2}}\left(y \mid x_{1}, x_{2}\right)=p_{Y \mid X_{2} X_{1}}\left(y \mid x_{2}, x_{1}\right),
$$

and

$$
\sum_{x_{1}, x_{2}, y} p_{X_{1}}\left(x_{1}\right) p_{X_{2}}\left(x_{2}\right) p_{Y \mid X_{1} X_{2}}\left(y \mid x_{1}, x_{2}\right)\left(y-x_{i}\right)^{2} \leq D_{2}, \text { for } i=1,2 .
$$

We denote $\mathcal{A}$ the set of all such feasible DMMCs.

\subsection{Probability of Error and Capacity}

In this paper, we only consider the case of detecting at least one colluder. An error occurs when the decoded two fingerprints do not match the fingerprints of the real colluders:

$$
\hat{M}_{1} \notin\left\{M_{1}, M_{2}\right\} \text { and } \hat{M}_{2} \notin\left\{M_{1}, M_{2}\right\} .
$$

The average probability of error for a deterministic code $\left(f_{N}, g_{N}\right)$ when a DMCC $p_{Y \mid X_{1} X_{2}}$ is in effect is denoted by $P_{e}\left(f_{N}, g_{N}, p_{Y \mid X_{1} X_{2}}\right)$. The minmax probability of error for the class of constrained DMCCs considered is given by

$$
P_{e, N}=\min _{\left(f_{N}, g_{N}\right)} \max _{p_{Y \mid X_{1} X_{2}}} P_{e}\left(f_{N}, g_{N}, p_{Y \mid X_{1} X_{2}}\right)
$$

DeFinition 2.4. A fingerprinting rate $R$ is achievable if there exists a code $\left(f_{N}, g_{N}\right)$ such that $|\mathcal{M}|=\left\lceil 2^{N R}\right\rceil$ and $P_{e, N} \rightarrow 0$ as $N \rightarrow \infty$.

DeFInITION 2.5. The capacity $C\left(D_{1}, D_{2}\right)$ is the supremum of all achievable rates.

\subsection{Known Results on Capacity Formulas}

Somekh-Baruch and Merhav ${ }^{6,7}$ derived capacity and error exponent formulas for private fingerprinting, and Wang and Moulin ${ }^{8}$ derived capacity and error exponent formulas for public fingerprinting. These results ${ }^{6-8}$ were derived for the case that the cover, fingerprinted, and forgery signals are from finite alphabets. But the results can be extended to the case of continuous alphabets. We use the following known formulas and derive the capacity for the case of a Gaussian cover signal and the MSE distortion. Capacity is infinite if the feasible set of attacks $\mathcal{A}$ is empty.

THEOREM 2.6. ${ }^{6}$ The capacity of the private fingerprinting game is given by

$$
C\left(D_{1}, D_{2}\right)=\max _{p_{X \mid S} \in \mathcal{Q}_{1}} \min _{p_{Y \mid X_{1} X_{2}} \in \mathcal{A}} \frac{1}{2} I\left(X_{1}, X_{2} ; Y \mid S\right),
$$

where $X_{1}$ is independent of $X_{2}$ given $S, p_{X \mid S}=p_{X_{1} \mid S}=p_{X_{2} \mid S}$, and $S \rightarrow\left(X_{1}, X_{2}\right) \rightarrow Y$ forms a Markov chain. That is, the joint pdf of $\left(S, X_{1}, X_{2}, Y\right)$ is given by

$$
p_{S X_{1} X_{2} Y}\left(s, x_{1}, x_{2}, y\right)=p_{S}(s) p_{X \mid S}\left(x_{1} \mid s\right) p_{X \mid S}\left(x_{2} \mid s\right) p_{Y \mid X_{1} X_{2}}\left(y \mid x_{1}, x_{2}\right) .
$$


THEOREM 2.7. ${ }^{8}$ The capacity of the public fingerprinting game is given by

$$
C\left(D_{1}, D_{2}\right)=\max _{p_{X U \mid S} \in \mathcal{Q}_{2}} \min _{p_{Y \mid X_{1} X_{2}} \in \mathcal{A}} \frac{1}{2}\left[I\left(U_{1}, U_{2} ; Y\right)-I\left(U_{1}, U_{2} ; S\right)\right],
$$

where $U_{1} \in \mathbb{R}$ and $U_{2} \in \mathbb{R}$ are auxiliary random variables, $\left(X_{1}, U_{1}\right)$ is independent of $\left(X_{2}, U_{2}\right)$ given $S$, $p_{X U \mid S}=p_{X_{1} U_{1} \mid S}=p_{X_{2} U_{2} \mid S}$, and $\left(S, U_{1}, U_{2}\right) \rightarrow\left(X_{1}, X_{2}\right) \rightarrow Y$ forms a Markov chain. That is, the joint pdf of $\left(S, U_{1}, U_{2}, X_{1}, X_{2}, Y\right)$ is given by

$$
p_{S U_{1} U_{2} X_{1} X_{2} Y}\left(s, u_{1}, u_{2}, x_{1}, x_{2}, y\right)=p_{S}(s) p_{X U \mid S}\left(x_{1}, u_{1} \mid s\right) p_{X U \mid S}\left(x_{2}, u_{2} \mid s\right) p_{Y \mid X_{1} X_{2}}\left(y \mid x_{1}, x_{2}\right) .
$$

\section{MAIN RESULTS}

THEOREM 3.1. For a Gaussian cover signal $S \sim \mathcal{N}\left(0, \sigma^{2}\right)$, the private Gaussian fingerprinting game capacity $C^{\text {Priv }}\left(D_{1}, D_{2}\right)$ derived from (8) is determined as follows:

1. $C^{\text {Priv }}\left(D_{1}, D_{2}\right)=0$ if $D_{2} \geq\left(\sqrt{D_{1}}+\sigma\right)^{2}$;

2. $C^{\text {Priv }}\left(D_{1}, D_{2}\right)=\infty$ if $D_{2}<\frac{1}{2} D_{1}$; and

3. if $\frac{1}{2} D_{1} \leq D_{2}<\left(\sqrt{D_{1}}+\sigma\right)^{2}$,

$$
C^{\text {Priv }}\left(D_{1}, D_{2}\right)=\max _{\tilde{a} \in\left[1-\sqrt{\frac{D_{1}}{\sigma^{2}}, 1+\sqrt{\frac{D_{1}}{\sigma^{2}}}}\right]} \frac{1}{4} \log \left[1+\frac{2 \sigma_{Z}^{2}\left(\tilde{a}^{2} \sigma^{2}+\sigma_{Z}^{2}-D_{2}\right)}{\left(2 \tilde{a}^{2} \sigma^{2}+\sigma_{Z}^{2}\right)\left(2 D_{2}-\sigma_{Z}^{2}\right)}\right],
$$

where

$$
\sigma_{Z}^{2}=D_{1}-(\tilde{a}-1)^{2} \sigma^{2}
$$

Denote the maximizing $\tilde{a}$ of (10) by a. The maxmin in (8) is achieved by the following fingerprinting channel:

$$
X_{1}=a S+Z_{1}, \quad X_{2}=a S+Z_{2},
$$

where $Z_{1} \sim \mathcal{N}\left(0, \sigma_{Z}^{2}\right)$ and $Z_{2} \sim \mathcal{N}\left(0, \sigma_{Z}^{2}\right)$ are independent of $S$; the optimal collusion attack channel is

$$
Y=b\left(X_{1}+X_{2}+W\right)
$$

where $W \sim \mathcal{N}\left(0, \sigma_{W}^{2}\right)$ is independent of $X_{1}$ and $X_{2}$,

$$
b=\frac{a^{2} \sigma^{2}+\sigma_{Z}^{2}-D_{2}}{2 a^{2} \sigma^{2}+\sigma_{Z}^{2}}
$$

and

$$
\sigma_{W}^{2}=\frac{\left(2 a^{2} \sigma^{2}+\sigma_{Z}^{2}\right)\left(2 D_{2}-\sigma_{Z}^{2}\right)}{a^{2} \sigma^{2}+\sigma_{Z}^{2}-D_{2}}
$$

TheOREM 3.2. For a Gaussian cover signal $S \sim \mathcal{N}\left(0, \sigma^{2}\right)$, the public Gaussian fingerprinting game capacity derived from (9) is the same as the private game capacity:

$$
C^{\text {Pub }}=C^{\text {Priv }}
$$

When $\frac{1}{2} D_{1} \leq D_{2}<\left(\sqrt{D_{1}}+\sigma\right)^{2}$, the optimal fingerprinting channel is

$$
X_{1}=a S+Z_{1}, \quad X_{2}=a S+Z_{2},
$$

and

$$
U_{1}=\alpha S+Z_{1}, \quad U_{2}=\alpha S+Z_{2}
$$


where $Z_{1} \sim \mathcal{N}\left(0, \sigma_{Z}^{2}\right)$ and $Z_{2} \sim \mathcal{N}\left(0, \sigma_{Z}^{2}\right)$ are independent of $S$, a is the maximizer of (10),

$$
\sigma_{Z}^{2}=D_{1}-(a-1)^{2} \sigma^{2}
$$

and

$$
\alpha=\frac{2 a \sigma_{Z}^{2}}{2 \sigma_{Z}^{2}+\sigma_{W}^{2}}
$$

the optimal collusion attack channel is

$$
Y=b\left(X_{1}+X_{2}+W\right),
$$

where $W \sim \mathcal{N}\left(0, \sigma_{W}^{2}\right)$ is independent of $X_{1}$ and $X_{2}$,

$$
b=\frac{a^{2} \sigma^{2}+\sigma_{Z}^{2}-D_{2}}{2 a^{2} \sigma^{2}+\sigma_{Z}^{2}},
$$

and

$$
\sigma_{W}^{2}=\frac{\left(2 a^{2} \sigma^{2}+\sigma_{Z}^{2}\right)\left(2 D_{2}-\sigma_{Z}^{2}\right)}{a^{2} \sigma^{2}+\sigma_{Z}^{2}-D_{2}}
$$

Remark 1: For the Gaussian cover signal $S$ and the MSE distortion, the achievable rate of reliable transmission (the decoder correctly decodes at least one colluder's fingerprint) is the same whether or not the cover signal is available to the decoder! The same observation has been made for the watermarking case, ${ }^{9}$ which is a single-user version of the fingerprinting game.

Remark 2: In the small distortion case, $\sigma^{2} \gg D_{1}, D_{2}$, when $\frac{1}{2} D_{1} \leq D_{2}<\left(\sqrt{D_{1}}+\sigma\right)^{2}$, we have the optimal parameters: $a^{*} \approx 1, \sigma_{Z}^{2 *} \approx D_{1}, b^{*} \approx \frac{1}{2}, \sigma_{W}^{2 *} \approx 2\left(2 D_{2}-D_{1}\right)$ and

$$
C^{\text {Priv }}=C^{\text {Pub }} \approx \frac{1}{4} \log \left[1+\frac{D_{1}}{2 D_{2}-D_{1}}\right] \text {. }
$$

Remark 3: Capacity is infinite if $D_{2}<\frac{1}{2} D_{1}$ because the feasible set $\mathcal{A}$ is empty in that case.

\section{SKETCH OF THE PROOF OF THEOREM 3.1}

The maximal value of $\mathrm{E} X^{2}$ over all feasible $p_{X \mid S}$ is $\left(\sqrt{D_{1}}+\sigma\right)^{2}$ and achieved by $X=a S$ when $a=1+\sqrt{\frac{D_{1}}{\sigma^{2}}}$. The colluders can simply let $Y=0$ if $D_{2} \geq \mathrm{E} X^{2}$, and the value of the cost function of (8) is 0 . Thus, the capacity $C^{\text {Priv }}$ is 0 if $D_{2} \geq\left(\sqrt{D_{1}}+\sigma\right)^{2}$.

The rest of Theorem 3.1, i.e. when $D_{2}<\left(\sqrt{D_{1}}+\sigma\right)^{2}$, is proved by showing that the capacity $C^{\text {Priv }}$ is lower bounded and at the same time upper bounded by the value given in the theorem.

\subsection{Proof of the Direct Part: Lower Bound of $C^{\text {Priv }}$}

We obtain a lower bound on $C^{\text {Priv }}$ by fixing a (not necessarily optimal) covert private fingerprinting channel and looking for the minimizing collusion attack channel for this specific fingerprinting channel.

Suppose that $X_{1}=a S+Z_{1}$ and $X_{2}=a S+Z_{2}$, where the cover signal $S$ and fingerprints $Z_{1} \sim \mathcal{N}\left(0, \sigma_{Z}^{2}\right)$, $Z_{2} \sim \mathcal{N}\left(0, \sigma_{Z}^{2}\right)$ are mutually independent, and

$$
\sigma_{Z}^{2}=D_{1}-(a-1)^{2} \sigma^{2} .
$$

Only when

$$
a \in\left[1-\sqrt{\frac{D_{1}}{\sigma^{2}}}, 1+\sqrt{\frac{D_{1}}{\sigma^{2}}}\right],
$$

the variance $\sigma_{Z}^{2}$ is greater than 0 and fingerprints are nonzero. 
Thus,

$$
\mathrm{E}\left(X_{1}-S\right)^{2}=\mathrm{E}\left(X_{2}-S\right)^{2}=(a-1)^{2} \sigma^{2}+\sigma_{Z}^{2}=D_{1},
$$

and the assumed covert private fingerprinting channel is feasible.

We have

$$
\mathrm{E} X_{1} X_{2}=a^{2} \sigma^{2}
$$

and

$$
\mathrm{E} X^{2}=\mathrm{E} X_{1,2}^{2}=a^{2} \sigma^{2}+\sigma_{Z}^{2}=D_{1}+(2 a-1) \sigma^{2} .
$$

In order for $\mathrm{E} X^{2} \geq 0$, i.e. $X_{1,2} \not \equiv 0$, it is required that

$$
a \geq \frac{1}{2}\left(1-\frac{D_{1}}{\sigma^{2}}\right) .
$$

Since $1-\sqrt{x} \geq \frac{1}{2}(1-x)$ for $x \geq 0$, the interval given by (12) suffices to specify the meaningful values of $a$ and (16) does not impose an additional constraint.

From the fairness condition $p_{Y \mid X_{1} X_{2}}=p_{Y \mid X_{2} X_{1}}$, we have $\mathrm{E} X Y=\mathrm{E} X_{1} Y=\mathrm{E} X_{2} Y$. Let

$$
\begin{gathered}
b=\frac{\mathrm{E} X Y}{\mathrm{E} X^{2}+\mathrm{E} X_{1} X_{2}}, \\
V=b^{-1} Y,
\end{gathered}
$$

and

$$
W=V-X_{1}-X_{2}
$$

Thus

$$
\begin{aligned}
Y & =b\left(X_{1}+X_{2}+W\right), \\
\mathrm{E} X W & =\mathrm{E} X_{1} W=\mathrm{E} X_{2} W=0,
\end{aligned}
$$

and a feasible DMCC needs to satisfy

$$
\mathrm{E}\left(Y-X_{1}\right)^{2}=\mathrm{E}\left(Y-X_{2}\right)^{2} \leq D_{2} .
$$

Let us denote $\sigma_{W}^{2}=\mathrm{E} W^{2}$, then from (17)

$$
\begin{aligned}
\sigma_{W}^{2} & \leq b^{-2}\left[D_{2}-(b-1)^{2} \mathrm{E} X^{2}-b^{2} \mathrm{E} X^{2}-2 b(b-1) a^{2} \sigma^{2}\right] \\
& =b^{-2}\left[D_{2}-\mathrm{E} X^{2}-2 b(b-1)\left(\mathrm{E} X^{2}+a^{2} \sigma^{2}\right)\right]
\end{aligned}
$$

We substitute all the above-defined random variables to the cost function of (8):

$$
\begin{aligned}
J=\frac{1}{2} I\left(X_{1}, X_{2} ; Y \mid S\right) & \stackrel{(a)}{=} \frac{1}{2} I\left(X_{1}, X_{2} ; V \mid S\right) \\
& =\frac{1}{2} I\left(a S+Z_{1}, a S+Z_{2} ; a S+Z_{1}+a S+Z_{2}+W \mid S\right) \\
& \stackrel{(b)}{=} \frac{1}{2} I\left(Z_{1}, Z_{2} ; Z_{1}+Z_{2}+W \mid S\right) \\
& =\frac{1}{2}\left[h\left(Z_{1}, Z_{2} \mid S\right)-h\left(Z_{1}+Z_{2}+W \mid S, Z_{1}, Z_{2}\right)\right] \\
& \stackrel{(c)}{\geq} \frac{1}{2}\left[h\left(Z_{1}, Z_{2}\right)-h\left(Z_{1}+Z_{2}+W \mid Z_{1}, Z_{2}\right)\right] \\
& =\frac{1}{2} I\left(Z_{1}, Z_{2} ; Z_{1}+Z_{2}+W\right) \\
& \stackrel{(d)}{\geq} \frac{1}{2} I\left(Z_{1}, Z_{2} ; Z_{1}+Z_{2}+W^{*}\right) \\
& =\frac{1}{2}\left[h\left(Z_{1}+Z_{2}+W^{*}\right)-h\left(W^{*}\right)\right] \\
& =\frac{1}{4} \log \left[1+\frac{2 \sigma_{Z}^{2}}{\sigma_{W}^{2}}\right]
\end{aligned}
$$


where (a) follows from the fact that $Y=b V$ is a deterministic function of $V$; (b) is valid because the mutual information is conditioned on $S$; (c) follows from the facts that $Z_{1}$ and $Z_{2}$ are independent of $S$ and conditioning decreases the entropy; $(\mathrm{d})$ is obtained because a Gaussian independent noise $W^{*} \sim \mathcal{N}\left(0, \sigma_{W}^{2}\right)$ minimizes the mutual information when the noise power is fixed at $\sigma_{W}^{2} \cdot{ }^{10}$

We substitute (11) and (18) into (19). Given $a$, the colluders search for the optimal $b$ and $\sigma_{W}^{2}$ to minimize the lower bound on $J$. Calculation shows that the optimal $b$ is

$$
b^{*}=\frac{a^{2} \sigma^{2}+\sigma_{Z}^{2}-D_{2}}{2 a^{2} \sigma^{2}+\sigma_{Z}^{2}},
$$

and the optimal $\sigma_{W}^{2}$ is

$$
\sigma_{W}^{2 *}=\frac{\left(2 a^{2} \sigma^{2}+\sigma_{Z}^{2}\right)\left(2 D_{2}-\sigma_{Z}^{2}\right)}{a^{2} \sigma^{2}+\sigma_{Z}^{2}-D_{2}} .
$$

Then we seek $a$ that minimizes (21) and therefore maximizes (19). Finally, the capacity $C^{\text {Priv }}$ is lower-bounded by

$$
C_{G} \triangleq \max _{a \in\left[1-\sqrt{\frac{\overline{D_{1}}}{\sigma^{2}}}, 1+\sqrt{\frac{D_{1}}{\sigma^{2}}}\right]} \frac{1}{4} \log \left[1+\frac{2 \sigma_{Z}^{2}\left(a^{2} \sigma^{2}+\sigma_{Z}^{2}-D_{2}\right)}{\left(2 a^{2} \sigma^{2}+\sigma_{Z}^{2}\right)\left(2 D_{2}-\sigma_{Z}^{2}\right)}\right] .
$$

Note that if $D_{2}<\frac{1}{2} D_{1}$, then when $a=1, \sigma_{Z}^{2}=D_{1}$ and $\sigma_{W}^{2}<0$, i.e., the set of feasible DMCCs is empty and hence the capacity is infinite.

\subsection{Proof of the Converse Part: $C^{\text {Priv }} \leq C_{G}$}

We now only need to consider $\frac{1}{2} D_{1} \leq D_{2}<\left(\sqrt{D_{1}}+\sigma\right)^{2}$. We obtain an upper bound on $C^{\text {Priv }}$ by fixing a (not necessarily optimal) collusion attack channel and looking for the maximizing fingerprinting channel for this specific collusion channel.

Let the collusion attack channel be $Y=b V=b\left(X_{1}+X_{2}+W\right)$, where $W \sim \mathcal{N}\left(0, \sigma_{W}^{2}\right)$ is independent of $X_{1}$ and $X_{2}$, and $b$ and $\sigma_{W}^{2}$ will be optimized subject to the distortion constraints:

$$
\mathrm{E}\left(Y-X_{1}\right)^{2}=(b-1)^{2} \mathrm{E} X_{1}^{2}+b^{2} \mathrm{E} X_{2}^{2}+2 b(b-1) \mathrm{E} X_{1} X_{2}+b^{2} \sigma_{W}^{2}=D_{2},
$$

and

$$
\mathrm{E}\left(Y-X_{2}\right)^{2}=(b-1)^{2} \mathrm{E} X_{2}^{2}+b^{2} \mathrm{E} X_{1}^{2}+2 b(b-1) \mathrm{E} X_{1} X_{2}+b^{2} \sigma_{W}^{2}=D_{2} .
$$

Let $Z_{1}=X_{1}-a S$ and $Z_{2}=X_{2}-a S$, where $Z_{1}$ and $Z_{2}$ have the same pdf since $p_{X \mid S}=p_{X_{1} \mid S}=p_{X_{2} \mid S}$, and $a$ is a parameter to be optimized. We denote $\sigma_{Z}^{2}=\mathrm{E} Z_{1}^{2}=\mathrm{E} Z_{2}^{2}$. A feasible covert private fingerprinting channel satisfies

$$
\mathrm{E}\left(X_{i}-S\right)^{2}=(a-1)^{2} \sigma^{2}+\sigma_{Z}^{2}+2(a-1) \mathrm{E} S Z_{i} \leq D_{1}
$$

for $i=1,2$.

Substituting all the above-defined random variables to the cost function of (8), we have

$$
\begin{aligned}
J=\frac{1}{2} I\left(X_{1}, X_{2} ; Y \mid S\right) & \stackrel{(a)}{=} \frac{1}{2} I\left(X_{1}, X_{2} ; V \mid S\right) \\
& =\frac{1}{2} I\left(X_{1}, X_{2} ; X_{1}+X_{2}+W \mid S\right) \\
& =\frac{1}{2}\left[h\left(X_{1}+X_{2}+W \mid S\right)-h\left(X_{1}+X_{2}+W \mid S, X_{1}, X_{2}\right)\right] \\
& \stackrel{(b)}{=} \frac{1}{2}\left[h\left(X_{1}+X_{2}+W \mid S\right)-h(W)\right] \\
& =\frac{1}{2}\left[h\left(Z_{1}+Z_{2}+W \mid S\right)-h(W)\right] \\
& \stackrel{(c)}{\leq} \frac{1}{2}\left[h\left(Z_{1}+Z_{2}+W\right)-h(W)\right]
\end{aligned}
$$




$$
\begin{aligned}
& \stackrel{(d)}{\leq} \frac{1}{2}\left[h\left(Z_{1}^{*}+Z_{2}^{*}+W\right)-h(W)\right] \\
& =\frac{1}{4} \log \left[1+\frac{2 \sigma_{Z}^{2}}{\sigma_{W}^{2}}\right]
\end{aligned}
$$

where (a) follows from the fact that $Y=b V$ is a deterministic function of $V$; (b) is valid because $W$ is independent of $S, X_{1}$, and $X_{2} ;$ (c) follows from the fact that conditioning decreases the entropy; (d) is obtained because Gaussian independent random variables $Z_{1}^{*} \sim \mathcal{N}\left(0, \sigma_{Z}^{2}\right)$ and $Z_{2}^{*} \sim \mathcal{N}\left(0, \sigma_{Z}^{2}\right)$ maximizes the entropy. ${ }^{10}$

Simple calculation shows that since $\frac{1}{2} D_{1} \leq D_{2}<\left(\sqrt{D_{1}}+\sigma\right)^{2}$, then $\sigma_{W}^{2} \geq 0$ and $\sigma_{Z}^{2}>0$ : the optimal $b$ is the same as shown in (20), the optimal $\sigma_{W}^{2}$ is the same as shown in (21), the optimal $\sigma_{Z}^{2}$ is given by

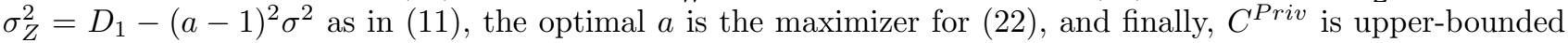
by $C_{G}$ shown in $(22)$.

\section{SKETCH OF THE PROOF OF THEOREM 3.2}

Since for the public Gaussian fingerprinting game, the cover signal $S$ is not available to the decoder, it follows naturally that $C^{\text {Pub }} \leq C^{\text {Priv }}$. We just need to prove that $C^{\text {Pub }} \geq C^{\text {Priv }}$ when $D_{2} \leq\left(\sqrt{D_{1}}+\sigma\right)^{2}$. Again, we obtain the lower bound by fixing a (not necessarily optimal) fingerprinting channel and looking for the minimizing collusion attack channel for this specific fingerprinting channel.

Assume that $X_{1}=a S+Z_{1}, X_{2}=a S+Z_{2}, U_{1}=\alpha S+Z_{1}$, and $U_{2}=\alpha S+Z_{2}$, where $Z_{1} \sim \mathcal{N}\left(0, \sigma_{Z}^{2}\right)$ and $Z_{2} \sim \mathcal{N}\left(0, \sigma_{Z}^{2}\right)$ are independent, and $a, \alpha$, and $\sigma_{Z}^{2}$ are to be optimized subject to

$$
\mathrm{E}\left(X_{1}-S\right)^{2}=\mathrm{E}\left(X_{2}-S\right)^{2}=(a-1)^{2} \sigma^{2}+\sigma_{Z}^{2}=D_{1} .
$$

Similarly to the steps we take in Section 4.1, we let

$$
\begin{gathered}
b=\frac{\mathrm{E} X Y}{\mathrm{E} X^{2}+\mathrm{E} X_{1} X_{2}}, \\
V=b^{-1} Y
\end{gathered}
$$

and

$$
W=V-X_{1}-X_{2}
$$

Thus

$$
\begin{aligned}
Y & =b\left(X_{1}+X_{2}+W\right), \\
\mathrm{E} X W & =\mathrm{E} X_{1} W=\mathrm{E} X_{2} W=0,
\end{aligned}
$$

and

$$
\mathrm{E}\left(Y-X_{1}\right)^{2}=\mathrm{E}\left(Y-X_{2}\right)^{2} \leq D_{2} .
$$

Let us denote $\sigma_{W}^{2}=\mathrm{E} W^{2}$, then

$$
\sigma_{W}^{2} \leq b^{-2}\left[D_{2}-(b-1)^{2} \mathrm{E} X^{2}-b^{2} \mathrm{E} X^{2}-2 b(b-1) a^{2} \sigma^{2}\right] .
$$

We substitute all the above-defined random variables to the cost function of (9):

$$
\begin{aligned}
J & =\frac{1}{2}\left[I\left(U_{1}, U_{2} ; Y\right)-I\left(U_{1}, U_{2} ; S\right)\right] \\
& \stackrel{(a)}{=} \frac{1}{2}\left[I\left(U_{1}, U_{2} ; V\right)-I\left(U_{1}, U_{2} ; S\right)\right] \\
& \stackrel{(b)}{\geq} \frac{1}{2}\left[I\left(U_{1}, U_{2} ; V^{*}\right)-I\left(U_{1}, U_{2} ; S\right)\right] \\
& \stackrel{(c)}{=} \frac{1}{2}\left[\log \frac{\left|K_{U_{1} U_{2}}\right|\left|K_{V^{*}}\right|}{\left|K_{U_{1} U_{2} V^{*}}\right|} \cdot \frac{\left|K_{U_{1} U_{2} S}\right|}{\left|K_{U_{1} U_{2}}\right|\left|K_{S}\right|}\right]
\end{aligned}
$$


where (a) follows from the fact that $Y=b V$ is a deterministic function of $V$; (b) is obtained because $V^{*}=$ $X_{1}+X_{2}+W^{*}$, where the Gaussian noise $W^{*} \sim \mathcal{N}\left(0, \sigma_{W}^{2}\right)$ is independent of $X_{1}$ and $X_{2}$, minimizes the mutual information when the noise power is fixed at $\sigma_{W}^{2}{ }^{10}$; (c) follows from the formula for the mutual information between two Gaussian random vectors

$$
I(\mathbf{X} ; \mathbf{Y})=\frac{1}{2} \log \frac{\left|K_{\mathbf{X}}\right|\left|K_{\mathbf{Y}}\right|}{\left|K_{\mathbf{X Y}}\right|}
$$

where $K_{\mathbf{X}}$ denotes the covariance matrix for the Gaussian random vector $\mathbf{X}$.

The encoder can maximize the right-hand side of (29), the lower bound on $J$, by selecting $\alpha$ to be

$$
\alpha^{*}=\frac{2 a \sigma_{Z}^{2}}{2 \sigma_{Z}^{2}+\sigma_{W}^{2}}
$$

so that

$$
J \geq \frac{1}{4} \log \left[1+\frac{2 \sigma_{Z}^{2}}{\sigma_{W}^{2}}\right] .
$$

The right-hand side of (30) is the same as the one in (19) for the private fingerprinting case. Consequently, the encoder/decoder applies the same set of optimal parameters as in the private game to maximize the right-hand side of (30), and the colluders do the same to minimize it. Finally, the capacity $C^{P u b}$ is lower bounded by $C_{G}$ defined in (22). Therefore, $C^{\text {Pub }}=C^{\text {Priv }}$.

\section{REFERENCES}

1. P. Moulin and A. Briassouli, "The Gaussian fingerprinting game," Proc. of the Conference on Information Sciences and Systems (CISS), Mar. 2002.

2. H. S. Stone, "Analysis of attacks on image watermarks with randomized coefficients," NEC TR 96-045, Princeton, NJ, 1996.

3. H. Zhao, M. Wu, Z. Wang and K. J. R. Liu, "Forensic analysis of nonlinear collusion attacks for multimedia fingerprinting," IEEE Trans. on Image Proc., vol. 14, no. 5, pp. 646-661, May 2005.

4. Z. Wang, M. Wu, H. Zhao, W. Trappe, and K. Liu, "Collusion resistance of multimedia fingerprinting using orthogonal modulation," IEEE Trans. on Image Proc., vol. 14, no. 6, pp. 804-821, Jun. 2005.

5. N. Kiyavash and P. Moulin, "A framework for optimizing nonlinear collusion attacks on fingerprinting systems," Proc. of the Conference on Information Sciences and Systems (CISS), Mar. 2006.

6. A. Somekh-Baruch and N. Merhav, "On the capacity game of private fingerprinting systems under collusion attacks," IEEE Trans. Inform. Theory, vol. 51, no. 3, pp. 884-899, Mar. 2005.

7. A. Somekh-Baruch and N. Merhav, "Achievable error exponents for the private fingerprinting game," submitted to IEEE Trans. Inform. Theory, Feb. 2005.

8. Y. Wang and P. Moulin, "Capacity and Random-Coding Error Exponent for Public Fingerprinting Game," Proc. of the International Symposium on Information Theory (ISIT), Jul. 2006.

9. P. Moulin and J. A. O'Sullivan, "Information-theoretic analysis of information hiding," IEEE Trans. on Information Theory, vol. 49, no. 3, pp. 563-593, Mar. 2003.

10. M. Pinsker, "Gaussian Sources," Probl. Inform. Transmission, vol. 14, pp.59-100, 1963. 